\section{LIF Density Measurement Calibration Using a Reference Cell}

M. T. Domonkos and G. J. Williams, Jr.

\author{
NASA John H. Glenn Research Center \\ Mail Stop 301-3 \\ 21000 Brookpark Road \\ Cleveland, $\mathrm{OH} 44135$
}

Flight qualification of ion thrusters typically requires testing on the order of 10,000 hours. Extensive knowledge of wear mechanisms and rates is necessary to establish design confidence prior to long duration tests. Consequently, real-time erosion rate measurements offer the potential both to reduce development costs and to enhance knowledge of the dependency of component wear on operating conditions. Several previous studies have used laserinduced fluorescence (LIF) to measure real-time, in situ erosion rates of ion thruster accelerator grids. Those studies provided only relative measurements of the erosion rate. In the present investigation, a molybdenum tube was resistively heated such that the evaporation rate yielded densities within the tube on the order of those expected from accelerator grid erosion. A pulsed UV laser was used to pump the ground state molybdenum at 345.64$\mathrm{nm}$, and the non-resonant fluorescence at 550-nm was collected using a bandpass filter and a photomultiplier tube or intensified CCD array. The sensitivity of the fluorescence was evaluated to determine the limitations of the calibration technique. The suitability of the diagnostic calibration technique was assessed for application to ion engine erosion rate measurements.

This is a preprint or reprint of a paper intended for presentation at a conference. Because changes may be made before formal publication, this is made available with the understanding that it will not be cited or reproduced without the permission of the author.

\section{ICOPS $_{2002}$}

International Conference on Plasma Science

\author{
Abstract Submitted for the 29th IEEE \\ International Conference on Plasma Science \\ May 26-30, 2002 \\ Banff, Alberta, Canada
}

Abstracts should be submitted by e-mail using this MS Word file.

Subject Topic_Plasma Diagnostics

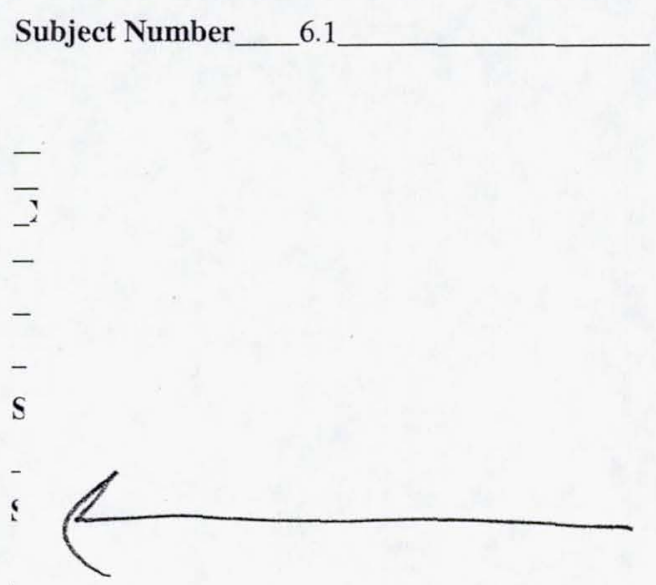

Province

Zip Code _ 44135

Country _USA

Phone 216-433-2164

Fax __216-433-2657

E-mail Matthew.T.Domonkos@grc.nasa.gov

Abstract must be received no later than

Jan. 18, 2002

e-mail to: icops2002@ee.ualberta.ca

If submitting by mail send original and two copies to:

\section{ICOPS2002}

Lee Grimard

423 Department of Physics

University of Alberta

Edmonton, AB Canada T6G 2J1 


\title{
LIF Density Measurement Calibration Using a Reference Cell
}

\author{
Matt Domonkos \\ NASA Glenn Research Center \\ Cleveland, $\mathrm{OH}$ \\ Matthew.T.Domonkos@grc.nasa.gov \\ George J. Williams, Jr. \\ OAl \\ Cleveland, $\mathrm{OH}$
}




\section{Outline}

- Background

- Fundamentals

- Description of Experimental Apparatus

- Density Distributions

- Application

- Conclusions 


\section{Ion Engines}
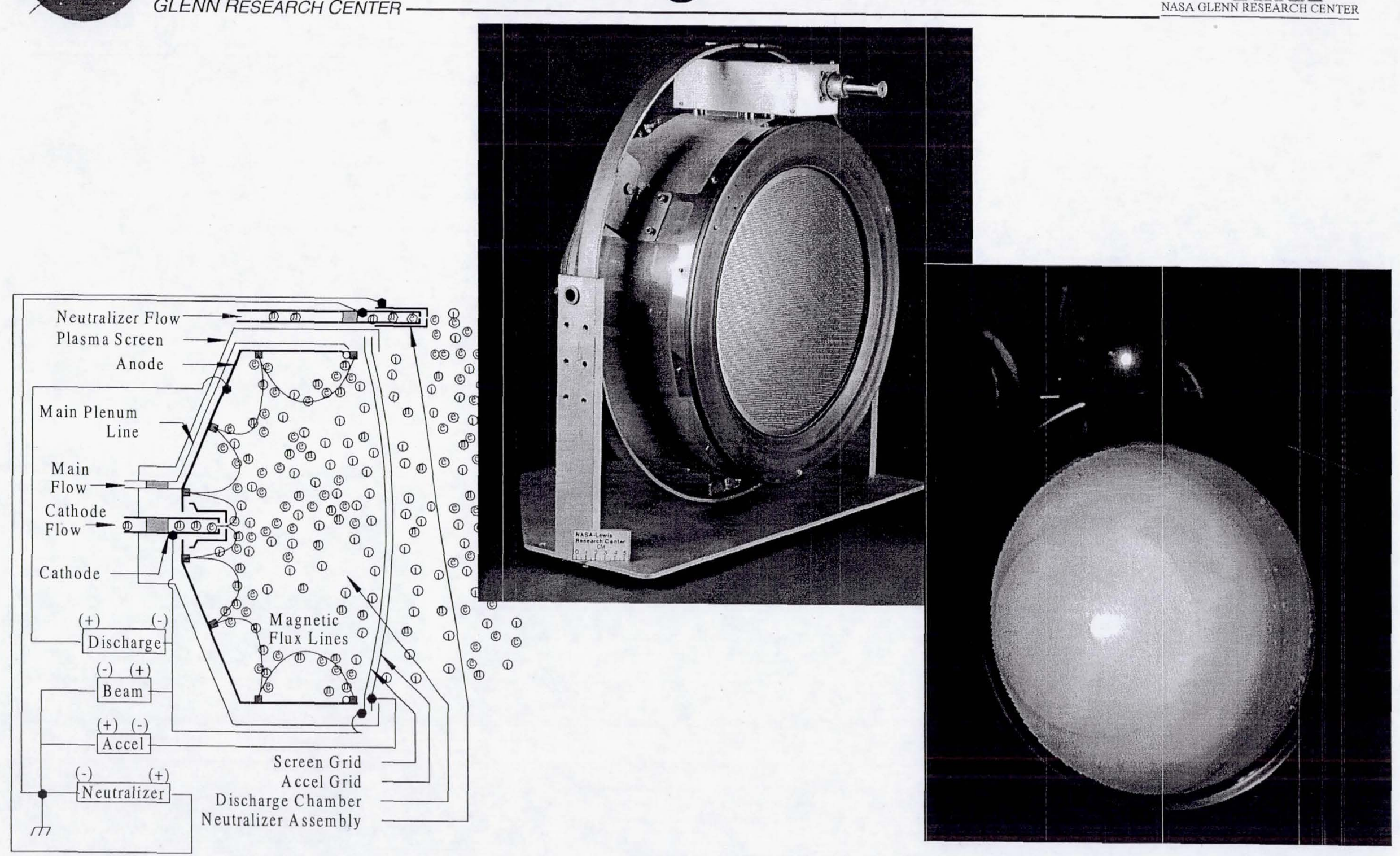


\section{Background}

\section{lon Engines}

- Ion engines for planetary and deep-space missions must operate from 10,000 to as much as 88,000 hours.

- The accelerator grid erodes under bombardment by the charge exchange plasma downstream of the thruster.

- The screen grid and discharge chamber elements are sputtered by near-threshold energy ions.

\section{Ion Engine Wear Testing}

- Historically, ion engines have been ground tested to at least their maximum required in-space propellant throughput.

- Few facilities exist which are capable of providing a suitable testing environment.

- Full life tests for ion engines are expensive and time consuming.

\section{Laser Induced Fluorescence}

- Laser induced fluorescence has been used to detect molybdenum as it is sputtered from the accelerator grid of an ion engine.

- The LIF intensity was correlated to the bias of the accelerator grid, however, these tests were done at potentials beyond the range used in NASA ion engines.

- The demonstrated LIF signal was a relative measure of the erosion.

\section{Density Calibration}

- The LIF signal may be calibrated by comparison to the signal obtained when interrogating a density standard.

- A reference cell has been fabricated based upon the vapor pressure of molybdenum. 


\section{Calibration Cell Fundamentals}

- The vapor pressure of metals is relatively well known - to within at least five percent.

- Create a cell of the target material with optical access and which may be heated to the relevant temperature.

- The density of the target atoms is related to the pressure through the ideal gas law for the vapor.

- For molybdenum, the evaporation rate yields the desired density at approximately $1962-\mathrm{K}$ to match $8,200 \mathrm{hr}$ wear test.

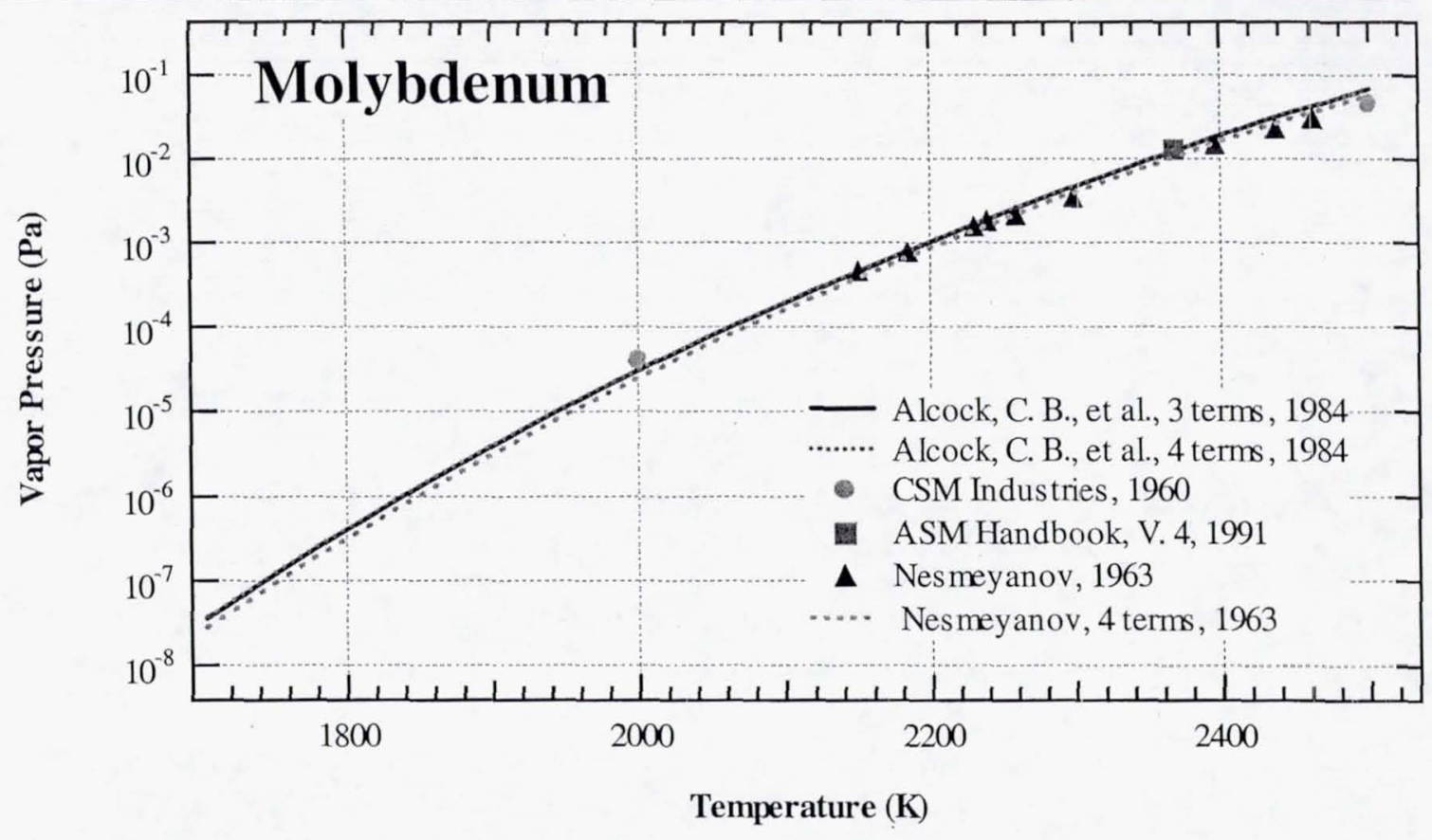

- Temperature gradients should be minimized, but this is difficult at the required temperatures.

- Can calculate the density distribution about the material for real temperature distribution and geometry.

- Cylindrical geometry chosen here. 


\section{Calibration Cell Schematic}

GLENN RESEARCH CENTER

\section{Alternate Scheme}

- High temperature thermocouples.

- Filter and PMT or intensified CCD for fluorescence collection.
Disappearing Filament

Pyrometer

Fluorescence Collection

Optics
Beam Steering

Mirrors

Tunable Laser Passes

Along Tube Axis

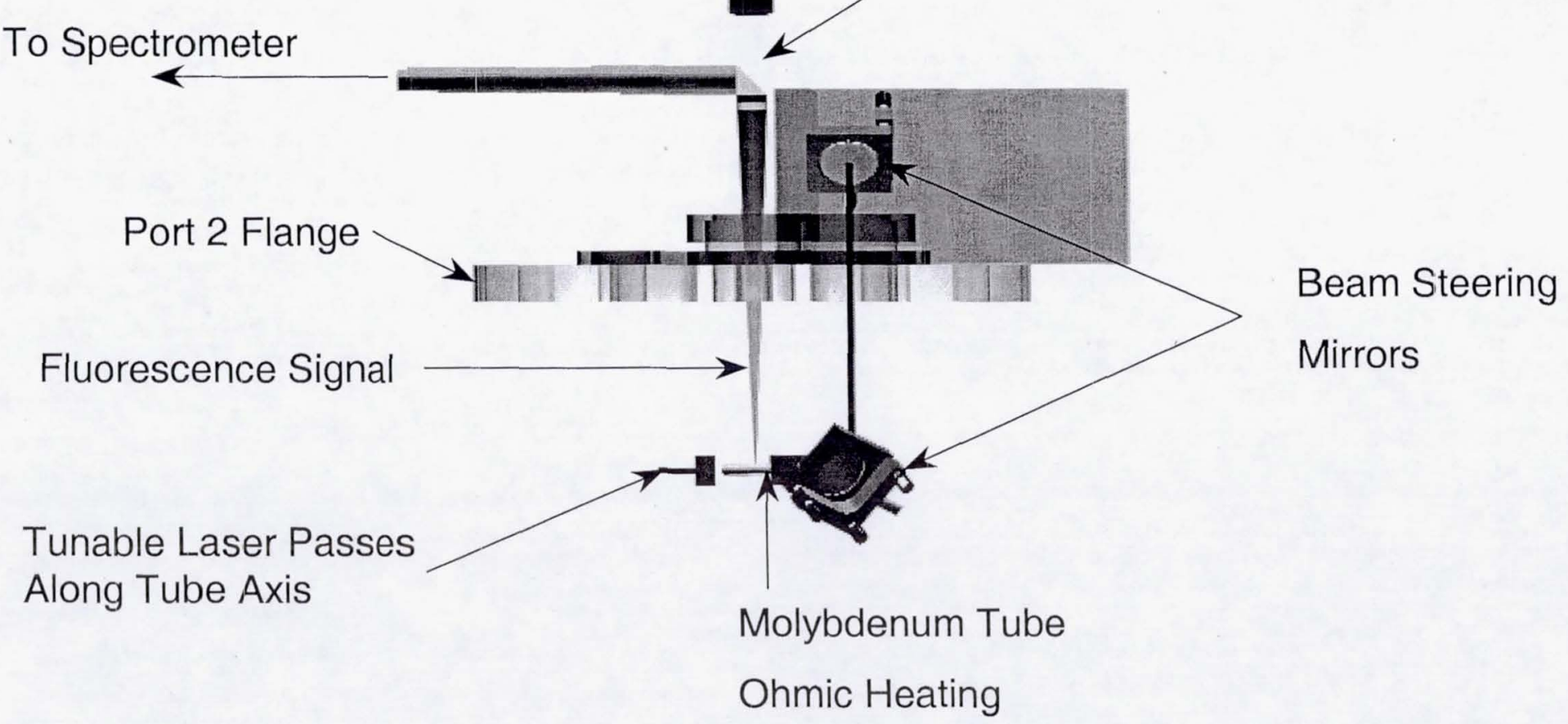




\section{Calibration Cell}
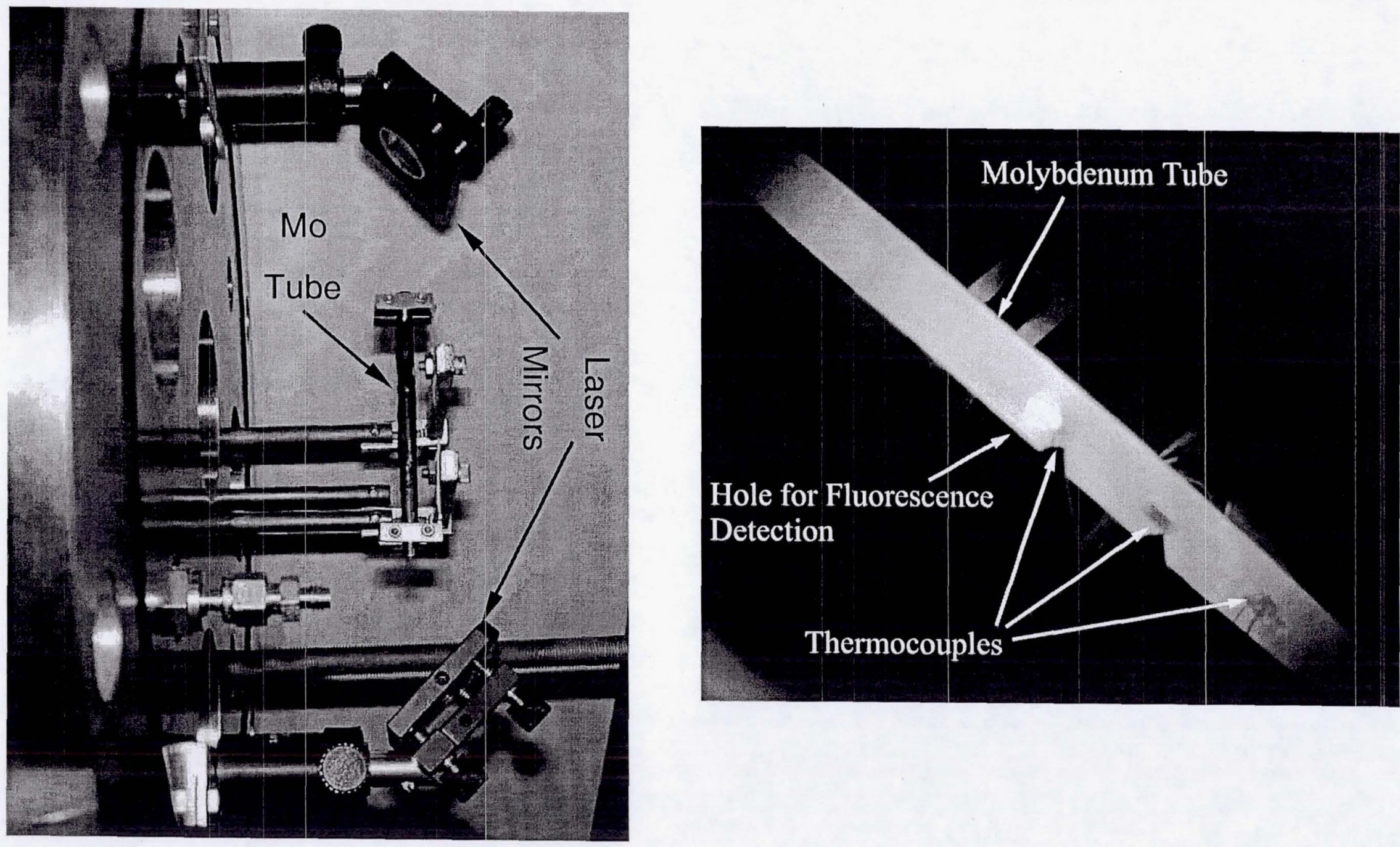


\section{Temperature Distribution and Vapor}

Pressure

GLENN RESEARCH CENTER

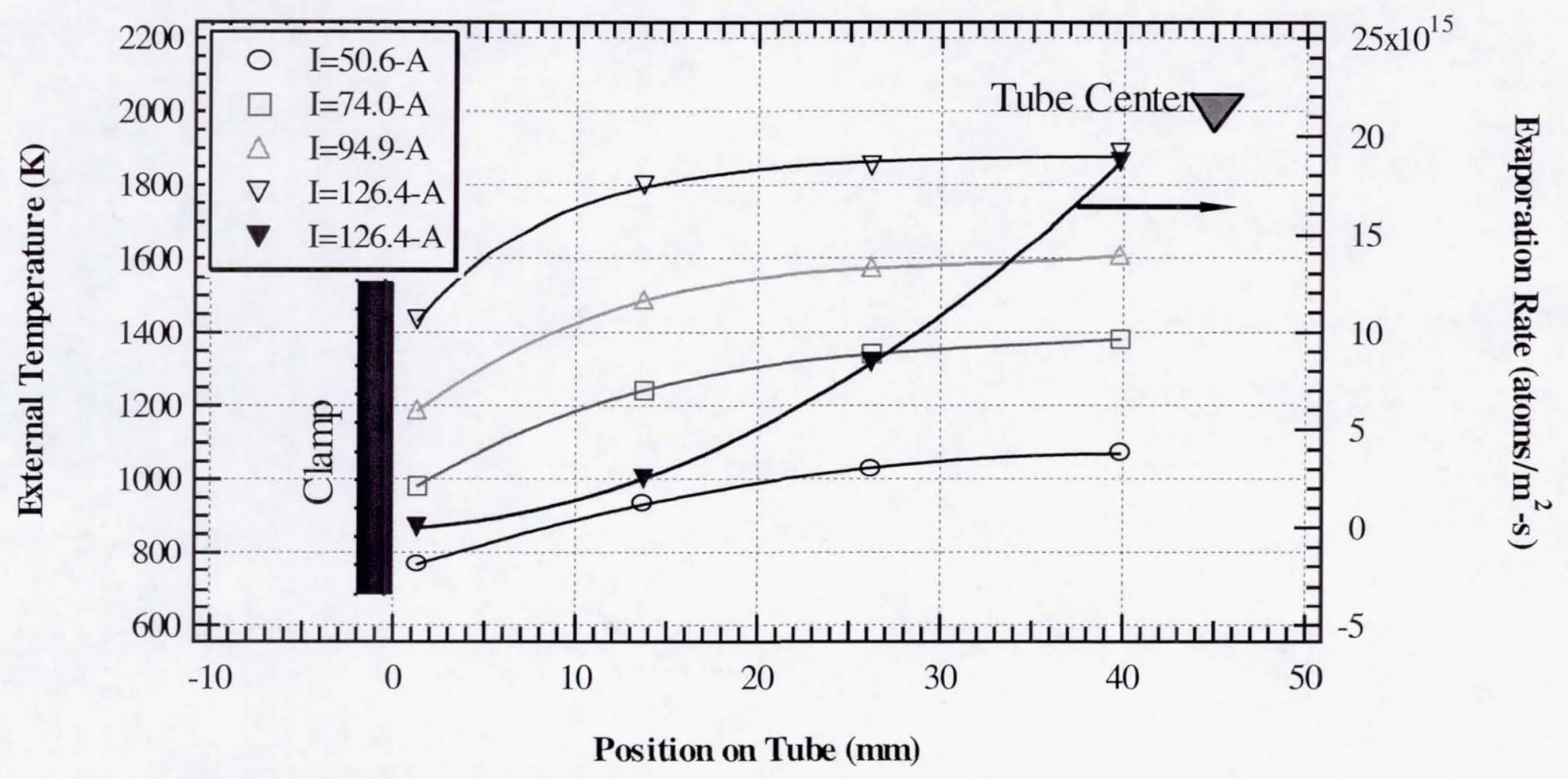

- Although the temperature gradient is approximately $20-\mathrm{K} / \mathrm{cm}$ near the center, becomes greater near the ends.

- Adds to non-uniformity.

- Evaporation rate varies by approximately a factor of 20 along the tube length.

- NEED A CALCULATION OF THE VAPOR DISTRIBUTION IN THE CELL. 


\section{Vapor Density Distribution}

GLENN RESEARCH CENTER

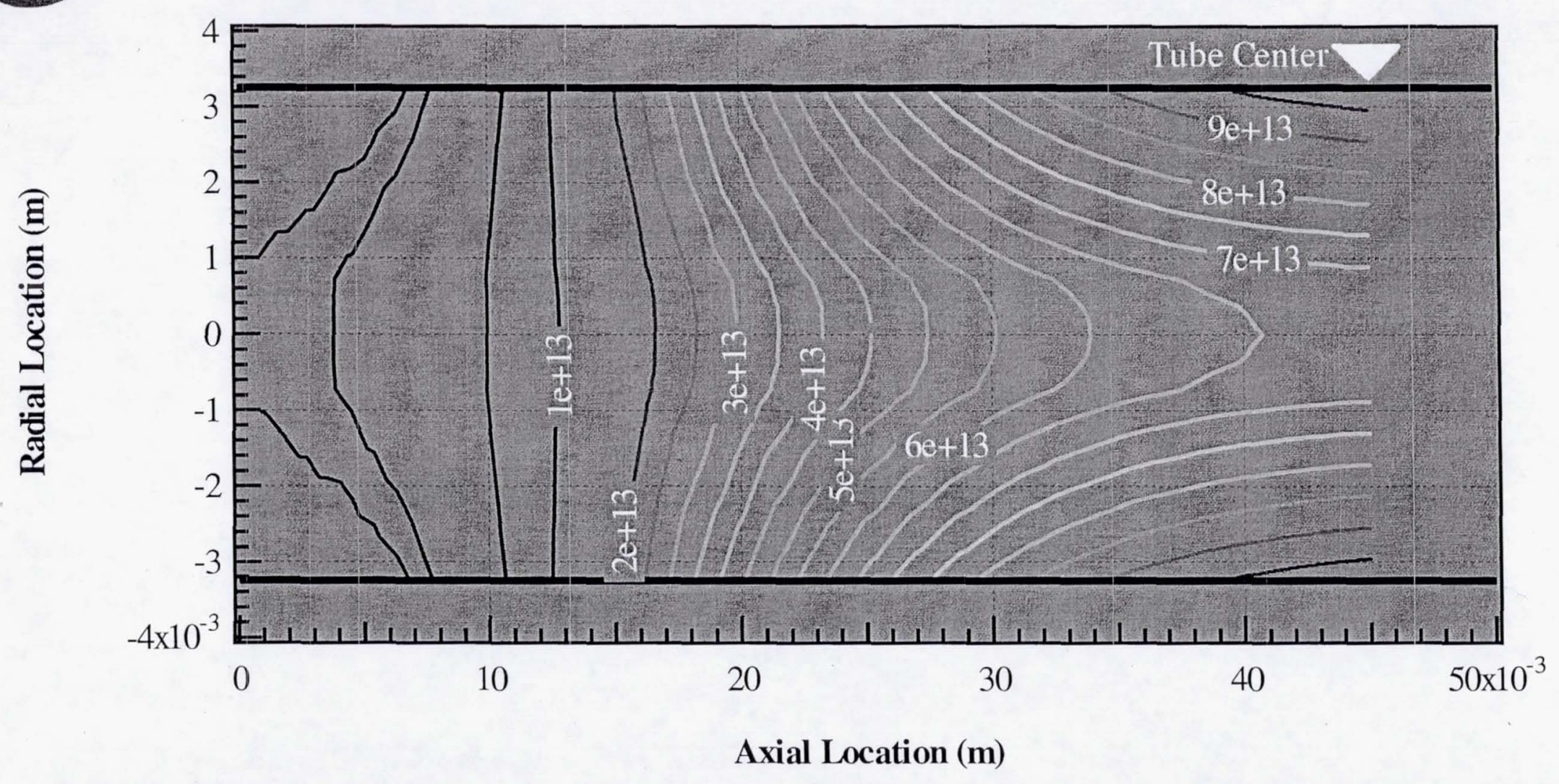

- Calculated assuming a hemispherical evaporative flux $\rightarrow$ can be solved for other flux distributions, but requires additional detail.

- Radial gradients near the center (interrogation region) are relatively severe.

- Calculation here is based on external tube temperature.

- Need internal temperature measurement for accuracy. 


\section{Error Analysis and Application}

GLENN RESEARCH CENTER

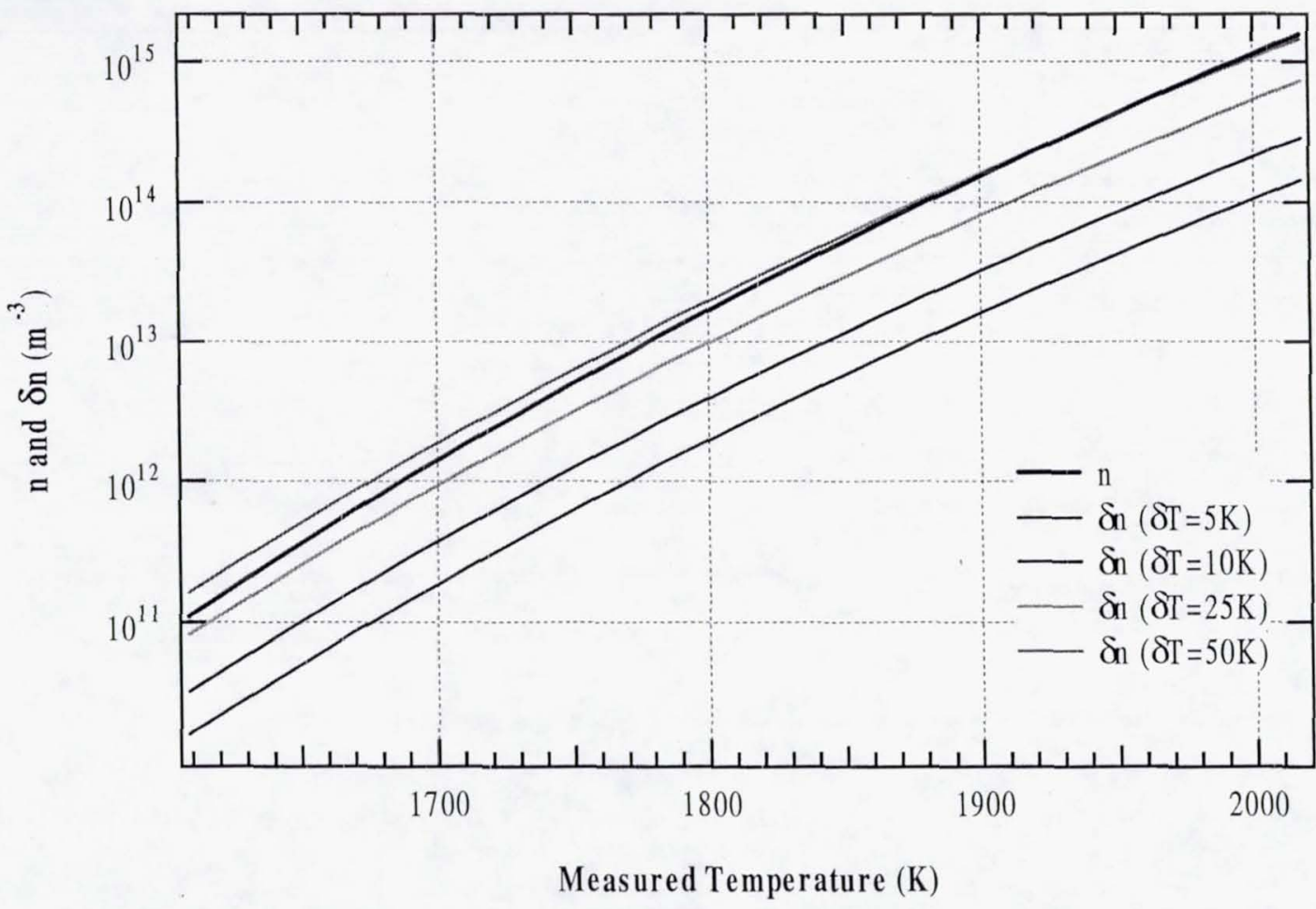

- A temperature error of $10-\mathrm{K}$ results in an error of 18 to 29 percent in density.

- Type $\mathrm{C}$ thermocouple rated for this range, but not NIST traceable.

- Error due to thermal contact and heat conduction may be significant. Requires examination.

\section{Application}

- This technique should be used in situ with the same laser and collection optics for use with a thruster.

- Calibrate over the expected range of densities - similar to any other calibration.

- The range expands with engine throttling capabilities. 


\section{Initial LIF Tests}

Tests with ICCD Detector

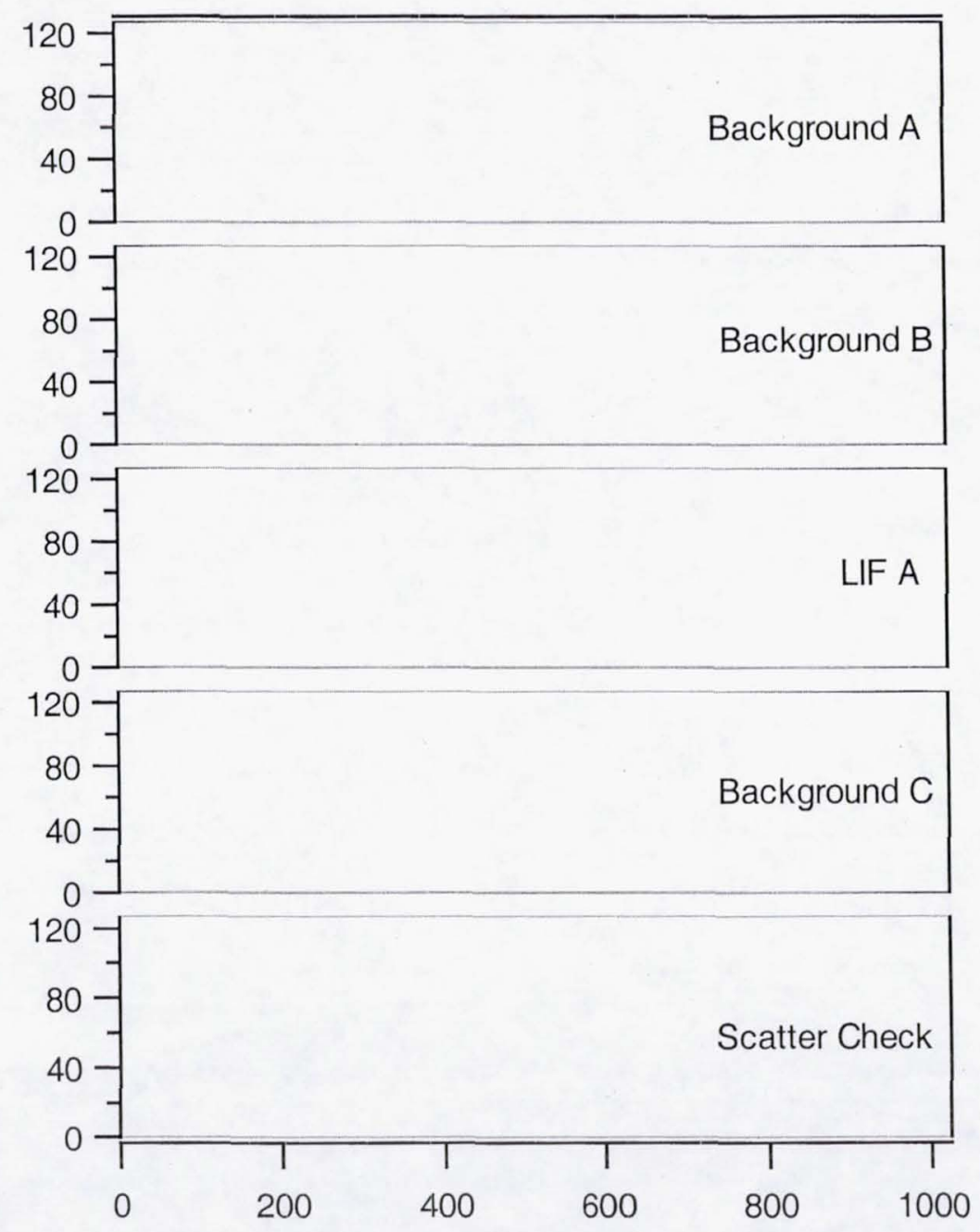

- ICCD:

- Focused on interrogation region.

- Gated operation with adjustable delay and width to capture fluorescence with minimal background accumulation.

- Filtered with 550-nm centered interference filter with a 3-nm FWHM.

- Triggering issues are currently limiting the duration of the gate pulse to several hundred nanoseconds.

- In this range, the background signal from the calibration cell approaches saturation in the ICCD.

- Alternate viewing approaches are being considered.

- Laser power is inadequate for LIF at temperatures tested so far (up to $1500 \mathrm{C}$ ). 


\section{Conclusions}

- A calibration density reference cell was developed to use with LIF to measure absolute vapor density.

- System calibration with a reference vapor density enables absolute measurement of erosion product density using LIF.

- Basis of operation discussed.

- Initial tests of the cell were conducted.

- A calculation of the vapor density distribution in the cell was presented.

- The error inherent to the reference cell was discussed.

- The application of the cell was discussed. 